\title{
Types of colorectal cancer and dysplasia associated with inflammatory bowel disease
}

\author{
Hisham Abdullah Almottowa ${ }^{1 *}$, Abdulmohsen Yaseer Alkhars², Maram Hussam Hassan ${ }^{3}$, \\ Hamad Adel Alhamad ${ }^{4}$, Saad Munawwikh Alshammari ${ }^{5}$, Anas Adel Bahamdeen ${ }^{6}$, \\ Tariq Mohammed Ladnah ${ }^{7}$, Yousef Thalib Alalyani ${ }^{7}$, Ahmed Taher Al binmaan, \\ Sultan Ali Alajam9, Ahmed Abdullah Alzooabi ${ }^{10}$
}

\author{
${ }^{1}$ Department of General Surgery, King Fahad General Hospital, Jeddah, Saudi Arabia \\ ${ }^{2}$ College of Medicine, King Faisal University, Al Ahsa, Saudi Arabia \\ ${ }^{3}$ College of Medicine, Arabian Gulf University, Manama, Bahrain \\ ${ }^{4}$ Department of General Surgery, King Hamad University Hospital, Busaiteen, Bahrain \\ ${ }^{5}$ Department of Pediatric Surgery, Maternity and Children Hospital, Hail, Saudi Arabia \\ ${ }^{6}$ Department of Otolaryngology, Madinah General Hospital, Medina, Saudi Arabia \\ ${ }^{7}$ College of Medicine, King Khalid University, Abha, Saudi Arabia \\ ${ }^{8}$ Alnuzha Primary Healthcare Center, Ministry of Health, Al Ahsa, Saudi Arabia \\ ${ }^{9}$ College of Medicine, Ibn Sina National College, Jeddah, Saudi Arabia \\ ${ }^{10}$ Department of General Surgery, King Fahad Specialist Hospital, Dammam, Saudi Arabia
}

Received: 22 December 2021

Accepted: 06 January 2022

\section{*Correspondence:}

Dr. Hisham Abdullah Almottowa,

E-mail: ahmadragab@gmail.com

Copyright: () the author(s), publisher and licensee Medip Academy. This is an open-access article distributed under the terms of the Creative Commons Attribution Non-Commercial License, which permits unrestricted non-commercial use, distribution, and reproduction in any medium, provided the original work is properly cited.

\begin{abstract}
Ulcerative colitis (UC) and Crohn's disease (CD) are two major inflammatory disorders of the intestinal wall collectively known as inflammatory bowel disease (IBD). Colorectal carcinoma (CRC) is the most significant and grave consequence of IBD and is preceded by dysplasia in majority of the cases. In this review we aim to discuss the various types of dysplasia found in patients with CRC due to IBD. A thorough literature search was conducted in online databases such as PubMed, Google Scholar, from which all studies published in the last ten years were included in this review. The major development in diagnostic procedures and visualization modalities have aided our understanding of dysplasia, which is now known to be the strongest predictor and marker for CRC development. However, the unpredictable behavior and progression of dysplasia still warrants vigilant surveillance. Dysplasia has been classified on histological characteristics using grades of dysplasia from 'negative for dysplasia' to 'high grade dysplasia'. On visibility via an endoscope from 'visible dysplasia' to 'invisible dysplasia' and macroscopic features of 'conventional dysplasia' and 'non-conventional dysplasia'. No single classification can be utilized to define the stage of dysplasia and more importantly predict its progression and outcome of CRC. Using evidence-based medicine an integrated classification expanding on a management algorithm must be formulated by a panel of experts to steer management of the disease. A multidisciplinary, tailored approach with a strong emphasis on regular and timely surveillance to ensure early detection of CRC can enhance quality of life and patient outcomes.
\end{abstract}

Keywords: IBD, Dysplasia, CRC, Classification 


\section{INTRODUCTION}

Inflammatory bowel disease (IBD) is a chronic, inflammatory disease of the intestinal system and manifests in two main pathophysiological forms namely $\mathrm{UC}$ and CD. The inflammation in UC is limited to the mucosal or submucosal layer only and usually affects the large intestine and rectum, whereas $\mathrm{CD}$ is characterized by transmural inflammation of the intestine affecting all the layers of the gut and affects any part of the entire gastrointestinal tract from mouth the perineal area. ${ }^{1-3}$ IBD has a relapsing and remitting course, whereby remission is a period of resolution of symptoms for at least 12 months, while a relapse is defined by a flare of symptoms confirmed by laboratory parameters and imaging in a patient diagnosed with IBD during a period of remission. ${ }^{2,4}$

IBD has a bimodal distribution and peaks in two age groups, once between 15 to 30 years of age, followed by 50 to 80 years of age. ${ }^{5,6}$ Incidence rates of $\mathrm{CD}$ was previously higher in females; however, an equal distribution of $\mathrm{CD}$ has developed over time, whereas an equal distribution among both genders for UC was always present. The highest prevalence of IBD can be found in the western region. The incidence rate of IBD is estimated to be 2.5-3 million in Europe and 1.5 million in North America. ${ }^{6}$ In the European Union, an estimated 176,000 new cases are diagnosed annually of which 53,000 are CD and 123,000 are UC. ${ }^{7}$ However, the incidence and prevalence of IBD has been steadily growing in other regions such as South-East Asia due to urbanization, a change in lifestyle and dietary habits of the local population. ${ }^{8}$

The most significant consequence of IBD is cancer of the colon and rectum or CRC. ${ }^{2}$ The risk of colorectal cancer is 2-6 times higher in patients with IBD as compared to the general population. ${ }^{6,7,9,10}$ The risk of development of CRC in patients with IBD increases with time to $1 \%$ at 10 years, $3 \%$ at 20 years and $7 \%$ at 30 years. ${ }^{11}$ In the AsiaPacific region, the prevalence of CRC due to IBD is $0.3 \%$ to $1.8 \% .^{8}$ Colorectal cancer accounts for $10-15 \%$ of deaths in people diagnosed with IBD. ${ }^{5-7,12,13}$ Patients suffering from IBD experience poor quality of life due to the chronicity of the disease, wide range of symptoms and their severity, need for life-long surveillance and the natural progression of the disease which is unpredictable. ${ }^{14}$

More than $90 \%$ of CRCs in IBD develop from dysplasia which is a well-defined neoplastic change in the epithelium without invasion into the Lamina Propria, remaining confined to the basement membrane. ${ }^{10,12,15,16}$ Dysplasia can be confirmed by histopathology of colorectal biopsies, characterized by a disruption of architecture and cytology (both nucleus and cytoplasm). Terms such as Intraepithelial Neoplasia (IEN) and Noninvasive neoplasia (NiN) are also used. ${ }^{12}$ As Dysplasia is a precancerous lesion, it can be the most reliable marker in patients with IBD as a precursor to CRC. ${ }^{12}$ In this review we will extensively discuss the types of dysplasia associated with CRC in patients with IBD.

\section{LITERATURE REVIEW}

A robust search was conducted from electronic databases such as PubMed, Medline, Google Scholar and cochrane library. To avoid missing potential studies, a further manual search for papers using terms 'IBD' ' $U C$ ' ' $C D$ ' 'Dysplasia' 'types' was done through Google Scholar. All studies in the last ten years were included in this review. Only studies discussing adult populations were reviewed. Only studies in English were included.

\section{DISCUSSION}

The pathophysiology of IBD and CRC is a wide spectrum of complex pathways involving environmental factors, immunological responses to inflammation and genetic alterations that cascade a series of events leading to the development of cancer. ${ }^{6}$ Some common genetic alterations that have been speculated are loss of heterozygosity $(\mathrm{LOH})$ in tumor suppressor genes such as p53 and adenomatous polyposis coli (APC). ${ }^{6,12}$ CRC in IBD can also occur due to chromosomal instability, microsatellite instability (MSI) and Deoxyribonucleic acid hypermethylation. MSI exhibits mutations in B-Raf proto-oncogene, serine/threonine kinase (BRAF) which are regulatory genes are early findings in IBD associated carcinogenesis.6,12,17 Immunological pathways induce inflammatory mediators such as cyclooxygenase-2 (COX2), cytokines interleukin-1 (IL-1), interleukin-6 (IL-6), tumor necrosis factor alpha (TNF- $\alpha$ ), and interferon gamma (IFN- $\gamma$ ). TNF- $\alpha$ leads to the production of reactive oxygen species (ROS) propagating oxidative stress further. Activation of oncogenic pathways such as nuclear factor Kappa light chain enhancer of activated B cells $(\mathrm{NF}-\kappa \beta)$ occurs as a result of oxidative stress. These immunological pathways are closely interlinked with molecular pathways and lead to CRC over time, extent, duration and severity of IBD. , $7,12,17$

$\mathrm{CD}$ and UC both have characteristic clinical features. In $\mathrm{UC}$, the primary symptom can be constipation, but the hallmark feature of UC is bloody diarrhea or stool mixed with blood and or mucus. ${ }^{3,18}$ Abdominal pain is confined to the left lower quadrant. ${ }^{3}$ Hematochezia, which is rectal bleeding with bowel movement can also occur. Sites of inflammation are limited to the rectum including the sigmoid and the descending colon. Patients with CD suffer from abdominal pain, loss of appetite, persistent or recurrent diarrhea, consequential weight loss. Symptoms of nausea, vomiting, dysphagia also occur. Patients also experience peri-umbilical abdominal pain and tenderness in the right lower quadrant causing patients to reduce their required caloric intake in order to avoid postprandial discomfort, and thus due to poor nutrition and impaired vitamin absorption patients become cachectic. In advanced stages of inflammation, abdominal cramping, 
vomiting, distention occur leading to strictures causing partial or complete intestinal obstruction. ${ }^{3}$ Fistulas and perianal disease are a hall mark of CD. ${ }^{18}$ Frequent sites of the intestinal wall affected by $\mathrm{CD}$ are terminal ileum and the colon where involved segments show a discontinuous pattern. Sites of distribution- CD 25\% have colitis, $25 \%$ have ileitis, $50 \%$ have ileocolitis. $^{2}$

The incidence of IBD is steadily increasing in the Arab world. According to a systematic review by Mosli et al UC is estimated to be 2.33 per 100,000 persons per year, whereas the incidence of $\mathrm{CD}$ is estimated to be 1.46 per 100,000 persons per year among the countries of Saudi Arabia, Egypt, United Arab Emirates (U.A.E), Kuwait, Bahrain, Lebanon and Oman. ${ }^{19}$ A retrospective study on histopathological profiles of colorectal biopsy samples was conducted in Saudi Arabia. Out of 684 biopsy samples, 217 confirmed histological findings for UC (31.7\%), whereas 22 cases of CD accounted for $3.2 \%$ of all colonic biopsy samples. Furthermore, UC affected males more (127) than females (97) with a male: female ratio of 1.2:1 and was found in patients between the ages of 12 to 92 years of age, with a mean age of 37.7 years of age. On the contrary CD was more common in females than males with a female: male ratio of $1.2: 1$ and was found in patients between 15 and 40 years of age, with a mean age of 27.1 years. ${ }^{20}$

Dysplasia has been widely accepted as a progenitor to CRC in patients with IBD. It is a highly significant determinant of further intervention and management of the disease. Advances in procedures such as biopsy and visualization techniques such as endoscopy have enhanced our understanding of dysplasia and its various types thus improving our approach and treatment rationale. Despite our improved understanding, various aspects of the development of dysplasia, it's behavior, sequence and progression towards neoplasia is not as well understood. Dysplasia can occur and develop in various presentations. Over the years classifications of dysplasia have been developed and amended based on histological findings, endoscopic appearance and macroscopic features. $^{21}$

\section{Histological classification of dysplasia}

The Vienna classification of dysplasia has five categories, and its types are described using histological criteria, which is a modification of the Riddell's classification of dysplasia which had four categories. ${ }^{10}$ Negative for dysplasia implies normal mucosa and refers to the regenerative nature of the epithelium. During active inflammation, the epithelium undergoes reactive changes mimicking atypical features of dysplasia. Maturation of surface epithelial nuclei towards luminal surface is the most characteristic feature of non-dysplastic, regenerating epithelium. ${ }^{10,12}$ Indefinite for Dysplasia The term is used during active inflammation and in effect atypical features of regenerating epithelium are occurring simultaneously, or when dysplasia is focal and limited to bases of crypts.
These features to make it difficult to distinguish regenerating epithelium from low grade dysplasia. Technical difficulties such as improper sampling and poor orientation of biopsy samples also cause difficulties in distinction. ${ }^{10,12,22}$

Low-grade dysplasia (LGD) are characterized by crowded glands with mild abnormalities in size and shape. The epithelium is pseudostratified and shows no nuclear maturation at the surface. The cytoplasm is hypereosinophilic and depleted of mucin. LGD has large cigar shaped hyperchromatic nuclei, however nuclear polarity is intact as they are limited in the basal half of the cell cytoplasm. Prominent mitotic figures are seen but atypical mitotic activity is rare. ${ }^{12,22}$

High-grade dysplasia (HGD) is characterized by a combination of cytological atypia and architectural abnormalities. Abnormalities in architecture with irregularities in the contours of crypts and intraluminal bridges called a 'cribriform pattern' are characteristic of HGD. However other abnormalities such as cystic changes, branching, tubular or villiform surface, complex crypt budding, or crowding also known as a back-to-back growth pattern are also common and reflect dysregulated cellular proliferation. Epithelial cells become pseudostratified, have a high nuclear/cytoplasmic ratio, loss of nuclear polarity and pleomorphism where elongated nuclei are present basally as well as apically. The enlarged nuclei have prominent nucleoli with increased and atypical mitotic activity. Nuclei may sometimes be round but prominent nucleoli is constant. ${ }^{12,22}$ According to the IBD dysplasia morphology study group, colorectal biopsies showing abnormalities in at least three crypts are confirmed for HGD. ${ }^{12,22}$ Invasive Neoplasia When neoplastic cells extend beyond the basement membrane of dysplastic crypts, it can be defined as a carcinoma. When cancerous cells penetrate the lamina propria or muscularis mucosae it is called intramucosal carcinoma whereas cancer cells when detected in the submucosal layer is called submucosal carcinoma. Invasive carcinoma in its early stages is characterized by small glands, neoplastic clusters, or cancer cells in isolation. Infiltrating glands with varied size and shape, with a possibility of intraluminal necrosis is evident. Periglandular desmoplasia is a reliable feature of carcinoma. ${ }^{12}$

Despite the histological grading, dysplastic changes do not progress as a sequence of events from LGD to HGD and eventually Carcinoma. According to a study by Ullman et al cancer may arise with no prior evidence of dysplasia. $^{12,17,23}$ Although this classification is widely used but it does not come without limitations. Distinguishing indefinite dysplasia from LGD and differentiating LGD from HGD can be challenging and it is essential to have more than one experienced histopathologist to comment on the dysplastic findings to avoid a misdiagnosis. Nevertheless, high inter-observer or intra-observer variability can lead to conflicting results. ${ }^{12}$ 


\section{Endoscopic classification of dysplasia}

The Paris classification categorizes dysplasia based on their visibility via an endoscope. They can be broadly classified into visible dysplasia where dysplasia is identified on targeted biopsies from lesions visualized during colonoscopies and invisible dysplasia where dysplasia is identified on random or non-targeted biopsies of colonic mucosa in the absence of a visible lesion. ${ }^{9,24}$ Visible dysplasia is of two types, Polypoid where the lesion protrudes from the mucosa into the lumen of the colon or rectum by $\geq 2.5 \mathrm{~mm}$ and non-polypoid, where the lesion is with a protrusion less than $2.5 \mathrm{~mm}$ or no protrusion above the mucosa., ${ }^{9,24}$ Polypoid is further classified into pedunculated where the lesion is attached to the mucosa by a stalk and sessile where the lesion is not attached by a stalk and the entire base of the lesion is contiguous with the mucosa. ${ }^{9,24}$ Non-polypoid can also be further classified into flat elevated lesions and flat lesions. Flat elevated lesions consist of superficial elevated which is a lesion with a protrusion but $<2.5 \mathrm{~mm}$ above the mucosa (0-IIa). Flat elevated lesion with a central depression $(0-\mathrm{IIa}+\mathrm{c})$ flat elevated lesion with a raised broad-based nodule $(0-$ IIa + Is). Flat lesions are lesions without protrusion above the mucosa and include flat mucosal change with no protrusion above the mucosa $(0-$ IIb), depressed a lesion below the level of the mucosa (0IIc) and excavated, a deep depression below the level of the mucosa (III)..$^{9,24}$ The surveillance for colorectal endoscopic neoplasia detection and management in IBD patients: international consensus recommendations (SCENIC) consortium made modifications to the Paris classification nomenclature of dysplasia with two more descriptors based on whether the lesion is endoscopically resectable or endoscopically unresectable. ${ }^{24}$ The SCENIC guideline defines an endoscopically resectable lesion in which distinct margins of the lesion are identifiable. On visual inspection, an endoscopic resection of the lesion is evident as a complete removal which is consistent with a histological examination of the resected specimen. Lastly, biopsy specimens taken from mucosa immediately adjacent to the resection site are free of dysplasia on histologic examination. ${ }^{9,24}$ Two more characteristics that could be used to describe dysplastic lesions are ulceration that is a fibrous base with depth within the lesion and on borders of the lesion which can be distinct when the borders of the lesion are discrete and can be distinguished from surrounding mucosa while Indistinct are when the borders of the lesion are not discrete and cannot be distinguished from surrounding mucosa. ${ }^{9,24}$

\section{Morphological classification of dysplasia}

Dysplasia has also been broadly classified into two main categories on the basis of its morphological criteria as conventional dysplasia and non-conventional dysplasia. Conventional dysplasia is defined on the similarities of histological features in dysplasia arising in IBD CRC and sporadic adenomas. ${ }^{4}$ They are adenomatous dysplasia which occurs in the vicinity of inflamed mucosa. The size and shape of glands are irregular surrounded by loose stroma and inflammatory cells. The glandular epithelium is pseudostratified, and the surface has a mixture of normal and dysplastic glands as the dysplasia begins from the bottom of the crypts extending to the surface. The epithelial nuclei is hyperchromatic and pleiomorphic which is elongated, penicillate with mitoses. Dystrophic goblet cells may also be present. Adenomatous dysplastic lesions may have a tubular appearance and are called tubular adenoma, or tubulovillous appearance and called tubulovillous adenoma. ${ }^{10,22}$ Villous dysplasia has a villous, hypermucinous appearance and is found in the proximity of colitis associated cancer, often reported to co-exist with carcinomas. Serrated dysplasia and the association with IBD have been reported in literature. Serrated dysplasia can be further classified as hyperplastic polyp (HP), sessile serrated adenoma (SSA) or polyp (SSP) with or without evidence of dysplasia and traditional serrated adenoma (TSA). ${ }^{10}$ Nuclei of serrated dysplastic lesions are round or oval and vesicular. According to Shen's series, 96\% of the serrated lesions in IBD are hyperplastic, the remaining are SSA/P while TSA are rare. However, the overall incidence of serrated dysplastic lesions in IBD is low. ${ }^{10}$ In a large retrospective study by a Centre in North America, patients with IBD undergoing routine colonoscopic surveillance were evaluated for serrated colorectal polyps diagnosed from 2000-2013. Out of 6602 patients diagnosed with IBD undergoing endoscopic surveillance, 78 serrated polyps endoscopically resected during the surveillance period were identified making the prevalence rate to $1.2 \%$. Serrated polyps without dysplasia were more commonly found in women, whereas serrated polyps with LGD or Indefinite dysplasia were more common in men. ${ }^{25}$ Driessen et al report that sessile serrated polyp (SSP) with LGD is more prevalent in colitis associated cancer. Similarly, Jackson et al reported that patients with IBD with sessile serrated adenoma (SSA) show an increased risk for visible dysplasia. A study by Rubio et al assessed all the histological phenotypes in 100 colectomy specimens contrasting with CRCs, of which 50 specimens were of IBD patients and 50 were non-IBD patients. Out of 50 specimens with IBD, dysplastic lesions were found in $76 \%$ of the IBD cases (38/50). Furthermore, out of 38 dysplastic lesions $52.3 \%$ were villous (20/38), $28.9 \%$ were serrated (11/38) $5.3 \%$ were tubular (2/38) while the remaining $13.2 \%$ were mixed $(5 / 38)$. Therefore, it was implied that of all the dysplastic lesions, serrated and villous were the most common non-invasive neoplastic lesions from which IBD carcinomas originate accounting for $81.2 \%$ (31/38). A significant difference between IBD patients and non-IBD patients was identified only for serrated dysplastic lesions. In IBD patients, serrated dysplastic lesions were $29 \%$ whereas only $3 \%$ in non-IBD patients as sporadic adenomas. ${ }^{26}$

A large number of new morphological characteristics and patterns have been identified and are collectively termed as non-conventional dysplasia. ${ }^{4}$ Despite their rarity most of these novel patterns have been recognized to have a 
high risk of developing into LGD or HGD more than conventional dysplasia, yet they have been reported in very few studies. ${ }^{4,27}$ According to a report by choi, nonconventional dysplasia has a more frequent association with neoplasia (38\%) as compared to conventional dysplasia (19\%). ${ }^{4}$ Non-conventional dysplasia has 5 subtypes hypermucinous dysplasia, crypt cell dysplasia (CCD), dysplasia with increased Paneth cell differentiation (DPD), goblet cell deficient dysplasia (GCD). ${ }^{4}$ However, some studies include sessile dysplasia with their three subtypes of sessile serrated lesion (SSL)like dysplasia, traditional serrated adenoma (TSA)-like dysplasia and an additional serrated dysplasia not otherwise specified (NOS) in categories of nonconventional dysplasia. ${ }^{4}$

Hypermucinous dysplasia account for $2 \%$ of all dysplasia occurring in patients with IBD. It is mostly found in patients with UC $(86 \%)$. Morphologically they exhibit a tubulovillous/villous architecture in which more than $50 \%$ of the lesion is lined by tall prominent mucinous cells. $^{4}$

On histological grading, features of low-grade dysplasia (LGD) can be found in the crypts however, prominent mucinous differentiation causes nuclear atypia to decrease towards the surface epithelium. ${ }^{4}$ On endoscopic appearance majority of hypermucinous dysplasia occur as a polypoid lesion, however up to $42 \%$ can be invisible dysplasia or a non-polypoid flat lesion. ${ }^{4}$ Hypermucinous dysplasia can occur as a 'pure type' or 'mixed type' in which it co-exists with either conventional dysplasia or with another non-conventional dysplastic Lesion, however, the hypermucinous component should comprise more than $50 \%$ of the lesion to be classified as a 'mixed type' hypermucinous dysplastic lesion. ${ }^{4}$ According to a review by Choi, hypermucinous dysplasia was in found in $42 \%$ of patients with IBD CRC. ${ }^{4}$ Similarly, another study by Andersen et al reports that $49 \%$ of low grade hypermucinous dysplastic lesion (19/39) were co-detected with 23\% HGD lesions (9/39) and $26 \%$ of adenocarcinomas (10/39) in previous sites of biopsy or the same colonic segments in mean Follow-up time of 11 months. ${ }^{4,27}$ Thus, it can be implied that hypermucinous dysplasia is a marker for neoplasia and despite its lowgrade morphology, it should be considered a high-risk lesion for more ominous lesions such as HGD and CRC. ${ }^{4}$ Crypt cell dysplasia (CCD) also known as dysplasia with terminal epithelial differentiation accounts for $4 \%$ of all dysplastic lesions in IBD patients. ${ }^{4}$ Histological findings exhibit by crypt cell dysplasia characterized by slightly enlarged hyperchromatic nuclei. ${ }^{4}$ The shape of the nuclei may be round to oval or slightly irregular, and crowded at the base of the crypts, where increased mitotic activity may also be found. The crypt cell dysplasia does not involve the surface epithelium and no significant architectural atypia is observed. ${ }^{4}$ Only a few scattered cells exhibit more enhanced nuclear enlargement with or without absence of nuclear polarity, no evidence of HGD has been reported. ${ }^{4}$ On endoscopic viewing, it presents as invisible dysplasia or a flat lesion (Non-polypoid), however when it does appear visible on endoscopic findings, it is described as mildly inflamed or erythematous, edematous or even friable and scarred lesions. ${ }^{4} \mathrm{CCD}$ is also associated with a high risk for carcinoma and can be considered a marker for advanced neoplasia. ${ }^{4}$ In a study by Lee et al out of 7 patients with CDD, 4 developed HGD (57\%) while 2 developed CRC $(28 \%)$ in the same segments of the colon with a mean follow-up time of 27 months. ${ }^{27}$ Thus, like hypermucinous dysplasia, CDD also has low grade morphology, patients with CDD lesions should be considered high risk for neoplasia. ${ }^{4}$ It is challenging to diagnose CDD solely from a histological standpoint due to its endoscopic findings, high interobserver variability and misdiagnosing most often as Indefinite Dysplasia, it has been suggested to raise suspicions among Gastroenterologists and pathologists for any form of crypt cell atypia and patients should be under vigilant surveillance via colonoscopies every 3-6 months. ${ }^{4}$ Dysplasia With increased Paneth cell differentiation (DPD) is the most common nonconventional dysplasia representing $51 \%$ nonconventional dysplasia and $13 \%$ of all dysplastic lesions in patients with IBD. ${ }^{4,27}$ Morphologically, DPD mostly presents as a polypoid lesion $(70 \%) .{ }^{4}$ The characteristic histological feature of DPD is increased Paneth cell differentiation in at least two contiguous crypts with dysplasia at two different foci. ${ }^{4}$ It exhibits a tubular architecture mostly lined by elongated, hyperchromatic nuclei involving both crypts and the epithelial surface. ${ }^{4} \mathrm{~A}$ striking feature is although Paneth cells can be found scattered in other types of Dysplasia, they are not found in multiple crypts or multiple foci. Furthermore, the same extent of Paneth cell differentiation is found in the adjacent non-dysplastic mucosa. ${ }^{4}$ As opposed to hypermucinous dysplasia and $\mathrm{CCD}$, the risk of neoplasia in DPD is much lower lesions. ${ }^{4}$ In the study by Lee et al, it was reported that neoplasia was only observed in $15 \%$ of DPD lesions. ${ }^{27}$

Goblet cell deficient dysplasia (GCD) represents 3\% of all dysplastic lesions in patients with IBD. ${ }^{4}$ Endoscopically, GCD often occurs as invisible dysplasia or as a flat non-polypoid lesion, however, if it presents as visible on endoscopic findings, it occurs as a polypoid lesion. ${ }^{4}$ Histologically, GCD is characterized by a complete absence or depletion of goblet cells, causing an eosinophilic cytoplasm. Eosinophilic luminal secretion is also a salient feature of GCD. ${ }^{4}$ A tubular architecture with LGD characteristics in both crypts and surface epithelial cells are observed. ${ }^{4}$ Despite features of LGD, GCD lesions are associated with neoplasia. ${ }^{4}$ The review by Choi narrates around 40\% GCD lesions are associated with HGD during diagnosis. ${ }^{4}$ Similarly, in the study by Lee et al, out of 17 GCD Lesions, 4 were co-detected with HGD $(24 \%)$ while 6 were co-detected with adenocarcinoma $(35 \%)$ in the same segments of the colon with a mean follow-up time of 13 months. ${ }^{27}$ Lastly, sessile dysplasias include three distinct subtypes, including SSL like dysplasia, TSA-like dysplasia, and 
serrated dysplasia NOS. ${ }^{4}$ Macroscopically serrated dysplastic lesions usually present as polypoid lesions. Histologically, SSL-like dysplasia is shows distorted serrated crypts with prominent basal crypt dilatation (i.e., dilated L- or inverted T-shaped crypts) at the interface with muscularis mucosa. TSA-like dysplasia exhibits a tubulovillous/villous architecture lined by tall columnar cells with intensely eosinophilic cytoplasm and ectopic crypts whereas Serrated dysplasia NOS does not show characteristic features of SSL-like dysplasia or TSA-like dysplasia. ${ }^{4}$ Serrated dysplasia can co-exist with either conventional dysplasia or another non-conventional subtype as a minor component, but to be distinguished as a specific serrated subtype, a serrated architecture should be the predominant feature representing $>50 \%$ of the lesion. ${ }^{4}$ Thus, it is understood that non-conventional dysplasia is commonly found in patients with IBD and have a strong association and causation of HGD, and even CRC which can be seen in long term follow ups. ${ }^{27} \mathrm{In}$ the study by Lee et al out of 168 patients with IBD, 317 dysplastic lesions were detected. Out of 317 dysplastic lesions, 78 lesions from 56 patients were nonconventional dysplasia, (33\%) whereas 239 lesions from 149 patients were conventional dysplasia. (89\%). From 78 non-conventional dysplastic lesions, 51\% were DPD, $18 \%$ were CCD, $13 \%$ were GCD, $9 \%$ were hypermucinous dysplasia, and serrated dysplasias both SSL and TSA-like accounted for $4 \%$ of all nonconventional dysplasias. ${ }^{27}$

One of the major limitations of this study is the wide array of classifications for dysplasia in patients with IBD based on its various forms and presentations. Furthermore, discrepancies among classifications have also been noted, where certain subtypes from one classification are allocated in a separate classification in other studies, which becomes very challenging to follow when comparing literature. Lastly as the progression of CRC in IBD has a prolonged course with long term follow ups, very few prospective studies on IBD CRC are found, hence a limitation on recent data regarding transformation of dysplasia and its various subtypes to CRC has been noted. It is recommended to conduct retrospective studies on IBD CRC and evaluate associated dysplasia including its various subtypes from histopathological samples. Furthermore, long term follows up studies on surveillance in patients with IBD should be conducted in which newly diagnosed dysplastic lesions and CRC should be evaluated and reported. Future research should also be conducted on the various types of dysplasia and the associated risk of each type with development of CRC.

\section{CONCLUSION}

IBD comprising of $\mathrm{UC}$ and $\mathrm{CD}$ are idiopathic, long standing inflammatory diseases of the intestinal system. Patients with IBD have a very high risk of developing CRC. More than half of CRC in IBD arise from dysplasia. Due to the various presentations, dysplastic lesions have been classified based on, histological characteristics, findings on endoscopy and macroscopic features. Certain classifications have their own limitations, and none can be used exclusively for establishing a diagnosis and further management. An evidence-based classification integrating all forms and presentations of dysplasia including its subtypes mandated by a panel of experts including gastroenterologists, histopathologists should be devised and implemented. A patient centered, multidisciplinary approach with lifestyle changes, pharmacotherapy and vigilant surveillance at regular intervals, must be ensued for earlier detection of dysplasia and CRC in patients with IBD thereby improving patient outcomes and quality of life.

Funding: No funding sources

Conflict of interest: None declared

Ethical approval: Not required

\section{REFERENCES}

1. Gajendran M, Loganathan P, Catinella AP, Hashash JG. A comprehensive review and update on Crohn's disease. Disease-A-Month. 2018;64(2):20-57.

2. Rogler G. Chronic ulcerative colitis and colorectal cancer. Cancer Letters. 2014;345(2):235-41.

3. Rohr M, Narasimhulu CA, Sharma D, et al. Inflammatory diseases of the gut. J Med Food. 2018;21(2):113-126.

4. Choi C-HR, Al Bakir I, Hart AL, Graham TA. Clonal evolution of colorectal cancer in IBD. Nature Rev Gastroenterol Hepatol. 2017;14(4):218-29.

5. Dyson JK, Rutter MD. Colorectal cancer in inflammatory bowel disease: what is the real magnitude of the risk? World J Gastroenterol. 2012;18(29):3839.

6. Mattar MC, Lough D, Pishvaian MJ, Charabaty A. Current management of inflammatory bowel disease and colorectal cancer. Gastrointestinal Cancer Res. 2011;4(2):53.

7. Keller D, Windsor A, Cohen R, Chand M. Colorectal cancer in inflammatory bowel disease: review of the evidence. Techniques Coloproctol. 2019;23(1):3-13.

8. Zhiqin $\mathrm{W}$, Palaniappan S, Ali RAR. Inflammatory bowel disease-related colorectal cancer in the AsiaPacific region: past, present, and future. Intestinal Res. 2014;12(3):194.

9. Khalid S, Abbass A, Khetpal N, Shen B, Navaneethan U. Endoscopic detection and resection of dysplasia in inflammatory bowel diseasetechniques with videos. Int $\mathbf{J}$ Colorectal Dis. 2019;34(4):569-80.

10. Svrcek M, Borralho Nunes P, Villanacci V, et al. Clinicopathological and molecular specificities of inflammatory bowel disease-related colorectal neoplastic lesions: the role of inflammation. J Crohn's Colitis. 2018;12(12):1486-98.

11. Saraiva S, Rosa I, Moleiro J, Da Silva JP, Fonseca R, Pereira AD. Dysplasia Surveillance in Inflammatory 
Bowel Disease: A Cohort Study. GE-Portuguese J Gastroenterol. 2021;28(2):97-105.

12. Mescoli C, Albertoni L, D’incá R, Rugge M. Dysplasia in inflammatory bowel diseases. Digestive Liver Dis. 2013;45(3):186-94.

13. Stidham RW, Higgins PD. Colorectal cancer in inflammatory bowel disease. Clin Colon Rectal Surg. 2018;31(03):168-78.

14. Azer SA. Overview of molecular pathways in inflammatory bowel disease associated with colorectal cancer development. Eur J Gastroenterol Hepatol. 2013;25(3):271-81.

15. Rutter MD, Riddell RH. Colorectal dysplasia in inflammatory bowel disease: a clinicopathologic perspective. Clin Gastroenterol Hepatol. 2014;12(3):359-67.

16. Soetikno R, Kaltenbach T, McQuaid KR. Paradigm shift in the surveillance and management of dysplasia in inflammatory bowel disease (West). Digestive Endoscopy. 2016;28(3):266-73.

17. Ibraheim H, Dhillon AS, Koumoutsos I, Gulati S, Hayee BH. Curriculum review: colorectal cancer surveillance and management of dysplasia in IBD. Frontline Gastroenterol. 2018;9(4):271-7.

18. Bernstein CN, Fried M, Krabshuis J. World Gastroenterology Organization Practice Guidelines for the diagnosis and management of IBD in 2010. Inflammatory Bowel Dis. 2010;16(1):112-24.

19. Mosli M, Alawadhi S, Hasan F, Abou Rached A, Sanai F, Danese S. Incidence, Prevalence, and Clinical Epidemiology of Inflammatory Bowel Disease in the Arab World: A Systematic Review and Meta-Analysis. Inflammatory Intestinal Dis. 2021:1-9.

20. Albasri AM. Histopathological profile of benign colorectal diseases in Al-Madinah region of Saudi Arabia. Asian Pacific J Cancer Prevention. 2014;15(18):7673-7.
21. Mark-Christensen A, Laurberg S, Haboubi N. Dysplasia in inflammatory bowel disease: historical review, critical histopathological analysis, and clinical implications. Inflammatory Bowel Dis. 2018;24(9):1895-903.

22. Driessen A, Geboes KP, Dewit O, Jouret-Mourin A. Dysplasia in inflammatory bowel disease. Colitis. 2018:141-54.

23. Huang LC, Merchea A. Dysplasia and cancer in inflammatory bowel disease. Surg Clin. 2017;97(3):627-39.

24. Laine L, Kaltenbach T, Barkun A. SCENIC international consensus statement on surveillance and management of dysplasia in inflammatory bowel disease. Gastrointestinal Endoscopy. 2015;81(3):489501.

25. Ko HM, Harpaz N, McBride RB. Serrated colorectal polyps in inflammatory bowel disease. Modern Pathol. 2015;28(12):1584-93.

26. Rubio CA, Befrits R, Jaramillo E, Nesi G, Amorosi A. Villous and serrated adenomatous growth bordering carcinomas in inflammatory bowel disease. Anticancer Res. 2000;20(6C):4761-4.

27. Lee H, Rabinovitch PS, Mattis AN, Lauwers GY, Choi WT. Non-conventional dysplasia in inflammatory bowel disease is more frequently associated with advanced neoplasia and aneuploidy than conventional dysplasia. Histopathology. 2021;78(6):814-30.

Cite this article as: Almottowa HA, Alkhars AY, Hassan MH, Alhamad HA, Alshammari SM, Bahamdeen AA et al. Types of colorectal cancer and dysplasia associated with inflammatory bowel disease. Int J Community Med Public Health 2022;9:1061-7. 